\title{
Diálogos sobre os sistemas de informação em saúde do trabalhador: relato de experiencia
}

\author{
Dialogues on health information systems worker: experience report
}

Diálogos sobre sistemas de información de salud trabajador: informe de experiencia

Vinícius Rodrigues de Oliveira ${ }^{1 *}$, Maria Luiza Santos Ferreira ${ }^{1}$, Ana Karoline Alves da Silva ${ }^{1}$, Maria Jeny de Sousa Oliveira ${ }^{1}$, Josefa lara Alves Bezerra ${ }^{1}$, Safira de Sousa Leite ${ }^{1}$, Milena Silva Ferreira ${ }^{1}$, Samyra Paula Lustoza Xavier ${ }^{1}$.

\section{RESUMO}

Objetivo: Relatar a experiência vivenciada na realização de um seminário para discussão da saúde do trabalhador com ênfase nos sistemas de informação em saúde. Relato de experiência: Trata-se de um relato de experiência baseado nas vivências do I Seminário de Saúde do Trabalhador: Sistemas de Informação em Saúde, ocorrido em novembro de 2019. Os organizadores do seminário foram alunos do oitavo semestre da graduação em enfermagem de uma Instituição Pública de Ensino Superior no interior do Ceará, motivados pela docente da disciplina Enfermagem no Processo de Cuidar em Saúde do Trabalhador. O evento contemplou vários temas importantes quando a saúde do trabalhador está em debate, dentre eles: riscos ocupacionais, manejo do sistema em saúde do trabalhador e fichas de notificação e comunicação de acidentes e/ou mortes no trabalho. Considerações finais: Por meio do Seminário de Saúde do Trabalhador pôde-se evidenciar a carência de diálogos voltados a saúde do trabalhador, bem como as lutas para a consolidação das ações nessa esfera.

Palavras-chave: Saúde do trabalhador, Sistemas de informação em saúde, Promoção da saúde.

\begin{abstract}
Objective: To report the experience of conducting a seminar to discuss worker health with emphasis on health information systems. Experience report:This is an experience report based on the experiences of the 1st Seminar on Occupational Health: Health Information Systems, which took place in November 2019. The seminar organizers were students of the eighth semester of the undergraduate nursing course at a Public Education Institution Higher education in the interior of Ceará, motivated by the teacher of the discipline Nursing in the Process of Care in Occupational Health. The event covered several important themes when the health of the worker is under debate, among them: occupational risks, management of the worker health system and notification and communication forms for accidents and / or deaths at work. Final considerations: Through the Workers 'Health Seminar, it was possible to highlight the lack of dialogues focused on workers' health, as well as the struggles to consolidate actions in this sphere.
\end{abstract}

Keywords: Occupational health, Health information systems, Health promotion.

\section{RESUMEN}

Objetivo: Informar sobre la experiencia de realizar un seminario para discutir la salud ocupacional con énfasis en los sistemas de información de salud. Informe de experiencia: Este es un informe de experiencia basado

${ }^{1}$ Universidade Regional do Cariri (URCA), Iguatu - CE. *E-mail: viniciurodriguesvro@gmail.com 
en las experiencias del 1er Seminario sobre Salud Ocupacional: Sistemas de Información de Salud, que tuvo lugar en noviembre de 2019. Los organizadores del seminario fueron estudiantes del octavo semestre del curso de pregrado de enfermería en una institución pública de educación superior en el interior de Ceará, motivados por el profesor de la disciplina Enfermería en el proceso de cuidado de la salud laboral. El evento cubrió varios temas importantes cuando se debate la salud del trabajador, entre ellos: riesgos laborales, gestión del sistema de salud del trabajador y formularios de notificación y comunicación para accidentes y / o muertes en el trabajo. Consideraciones finales: A través del Seminario de Salud de los Trabajadores, fue posible resaltar la falta de diálogos centrados en la salud de los trabajadores, así como las luchas para consolidar las acciones en esta esfera.

Palabras clave: Salud ocupacional, Sistemas de información en salud, Promoción de la salud.

\section{INTRODUÇÃO}

O trabalho se configura como um conjunto de ações que necessitam de esforços dos indivíduos e que tem a capacidade de motivar e alterar as condições de viver, adoecer e morrer dos sujeitos sendo, portanto, um dos principais determinantes sociais que influenciam no processo de saúde-doença. Partindo desse pressuposto, a Saúde do Trabalhador (ST) se designa por uma abordagem multidisciplinar e intersetorial das práticas, com foco na promoção da saúde, prevenção de agravos e assistência curativa ao trabalhador, apresentando como eixos basilares, as causas ou os determinantes dos agravos, a exposição aos riscos, os danos ou as consequências destes (ROLOFF DIT, et al., 2016).

Nessa perspectiva, Vasconcellos LC e Aguiar L (2017) trazem que, na esfera do Sistema Único de Saúde (SUS), um dos campos de atuação que mais tem obstáculos para contornar e consolidar sua efetiva implantação é a ST e essa conjuntura está atrelado ao fato do SUS ser uma política de bem-estar social abrigada em um Estado de 'bem-estar do mercado' que coloca a ST no centro de um debate políticonormativo, ou seja, o que está estabelecido nas leis, normas e regras não tem sido efetivado de acordo com a realidade, e isso acaba explicando o desmedido resultado de adoecimento e morte no trabalho que a saúde pública ainda não se tem um dimensionamento.

Os agravos que acometem os trabalhadores, estão relacionados com as atividades profissionais que exercem, ou por condições perigosas existentes no seu trabalho (FRAZ EC e CARGNIN MCS, 2018). O Acidente de Trabalho (AT) é definindo pelo Ministério da Saúde (MS) como um episódio inesperado proveniente da atividade laboral, independentemente da situação empregatícia e previdenciária do trabalhador acometido, e que ocasiona prejuízos à saúde, imediatos ou a longo prazo, gerando lesão corporal ou distúrbio funcional que pode causar a morte, a perda ou a redução, inalterável ou temporária, da capacidade desenvolver o trabalho (BRASIL, 2006).

Os AT são vistos como sérios problemas socioeconômicos e de saúde pública. A Organização Internacional do Trabalho (OIT) aponta que, cerca de 4\% do Produto Interno Bruto (PIB) global acaba sendo convertido em gastos relacionados aos acidentes e doenças do trabalho (ILO, 2015). Em torno de 2,3 milhões de pessoas no mundo morrem a cada ano em virtude desses danos.

Além dos custos financeiros e sociais, os AT respondem por grande parcela dos procedimentos realizados em unidades de média e alta complexidade. Pouco mais de $25 \%$ das lesões por causas externas atendidas em serviços de emergência no Brasil são decorrentes de acidentes de trabalho. Apesar da sua relevância epidemiológica, o conhecimento da magnitude do problema no país ainda é subestimado (FERREIRA MJM, et al., 2017).

É importante que haja as notificações da totalidade dos AT, ou seja, a alimentação efetiva dos Sistemas de Informação, pois são indispensáveis para a efetivação de ações competentes de Vigilância em Saúde do Trabalhador e policiamento dos locais de trabalho. No entanto são vários os motivos que colaboram para a subnotificação dos AT no contexto da saúde pública, dentre eles: a desintegração dos sistemas de informação, principalmente na área da ST; a escassa experiência dos profissionais em referência aos 
instrumentos e comportamentos para notificação; o grande contingente de trabalhadores empregados sem um elo estável, ocasionando uma elevada alternância nos serviços de saúde; e a escassez de práticas de educação de forma contínua (LIMA RKS, et al., 2018).

Os sistemas de informação de instituições previdenciárias são os mais utilizados para um melhor acompanhamento, como também para estudos desses agravos que podem acometer a ST, já que, geralmente se baseiam na identificação do nexo ocupacional requerido para privilégios de compensação requeridos. Há a necessidade de uma precisão desses registros e informações para um apropriado planejamento e gestão (BATISTA AG, et al., 2019).

Nesse contexto, é de fundamental importância que os trabalhadores em saúde conheçam e se apropriem dos Sistemas de Informação voltados a ST para que possam contribuir na formulação de estratégias que diminuam os agravos existentes, contribuindo assim, na garantia de melhores condições de vida, trabalho e saúde desse público. Assim, esse estudo tem como objetivo relatar a experiência vivenciada na realização de um seminário para discussão da saúde do trabalhador com ênfase nos sistemas de informação em saúde.

\section{RELATO DE EXPERIÊNCIA}

Trata-se de um relato de experiência baseado nas vivências do I Seminário de Saúde do Trabalhador: Sistemas de Informação em Saúde, ocorrido em novembro de 2019. Os organizadores do seminário foram alunos do oitavo semestre da graduação em enfermagem de uma Instituição Pública de Ensino Superior no interior do Ceará, motivados pela docente da disciplina Enfermagem no Processo de Cuidar em Saúde do Trabalhador.

O evento contemplou vários temas importantes quando a ST está em debate, dentre eles: riscos ocupacionais, manejo do sistema em ST e fichas de notificação e comunicação de acidentes e/ou mortes no trabalho. É mister ressaltar que o evento aconteceu em um momento oportuno, pois favoreceu o debate acerca da então recente atualização sobre a caracterização dos acidentes de trajeto.

A explanação desses temas foi conduzida pelos estudantes organizadores do evento e posteriormente por profissionais convidados, dentre eles um técnico em segurança do trabalho e uma psicóloga, ambos funcionários do Centro de Referência em Saúde do Trabalhador (CEREST) que dá cobertura a região centrosul do Ceará. Os palestrantes mostraram as suas atribuições, considerando as especificidades de cada profissão, a forma de organização do trabalho e alguns exemplos de atendimentos feitos durante os 12 anos em que vêm atuando na instituição, reafirmando a necessidade de se discutir sobre as fichas e os sistemas de notificação.

Após a apresentação do conteúdo abriu-se espaço para indagações do público presente, que estavam distribuídos entre estudantes da graduação em enfermagem e trabalhadores da instituição sediadora, somando um total de 67 participantes.

Durante o momento de explanação foi possível evidenciar os inúmeros desafios no cuidado à saúde do trabalhador além de provocar reflexões necessárias acerca do papel dos profissionais de saúde e a importância da notificação dos agravos e mortes no trabalho. Sendo este último verificado por meio das perguntas do público a dificuldade no preenchimento das fichas de notificação e principalmente na identificação de quais agravos notificar.

Tais discussões evidenciaram, em partes, dificuldades que já eram apontadas pela literatura, como por exemplo, a subnotificação de morte ou acidentes de trabalho, fato que ocorre, muitas vezes, devido a inaptidão do profissional para preencher essas fichas.

Outro ponto sinalizado durante o seminário referiu-se a baixa adesão dos profissionais em momentos de educação continuada, principalmente na área de ST, que tem grandes reveses a serem superados. Esse aspecto reafirma ainda mais a relevância e o impacto que eventos desse tipo sejam realizados como forma de capacitar e sensibilizar os profissionais de saúde para atuarem na área.

Além da abordagem a ST, o evento propiciou aos acadêmicos de Enfermagem envolvidos, a vivência de experiências importantes, favorecendo o desenvolvimento de competências profissionais, como o trabalho 
em equipe, a comunicação, a parceria, a liderança, o planejamento, a implementação, dentre outros aspectos que trouxeram uma significativa contribuição para a formação acadêmico-profissional, especialmente, no aprimoramento das competências voltadas a ST.

\section{DISCUSSÃO}

A idealização do evento deu-se a partir do desejo de ampliar conhecimentos acerca da vigilância em saúde e epidemiologia relacionadas a ST com ênfase nos sistemas de informação em saúde, de modo que favorecesse a reflexão crítica, a aprendizagem significativa, coletiva e interdisciplinar.

Entender a importância da notificação dos acidentes de trabalho é a peça-chave para o controle e prevenção de agravos em ST. Notificar agravos e acidentes pertinentes ao trabalho, é uma importante ferramenta para delinear programas e políticas no âmbito da saúde do trabalhador, no entanto, muitos profissionais não realizam esses registros. Essa situação, muitas vezes, relaciona-se com a ausência de processos formativos que capacite os profissionais para tal prática, dificultando a compreensão das dimensões que envolvem a saúde do indivíduo e sua ocupação.

Esse cenário corrobora com o estudo de Pedroso HC e Gonçalves CGO (2016), que evidencia a inexistência de envolvimento dos profissionais da saúde com as bases conceituais de saúde do trabalhador, isso os fazem ignorar que o trabalho tem forte influência no processo de saúde doença das pessoas, e que o planejamento de intervenções no espaço laboral, são necessárias.

O preenchimento adequado dos formulários de notificação de agravos a ST são importantes, pois a partir desses dados são traçadas estratégias de prevenção de doenças e acidentes de trabalho, além da realização de ações que visem a reabilitação e promoção da saúde, as quais devem ser desenvolvidas por uma equipe multidisciplinar (FRANZ EC e CARGNIN MCS, 2017).

Vê-se, portanto, que é necessário que haja uma identificação e notificação dos problemas de saúde relacionados às atividades de trabalho e riscos laborais, pois desse modo, torna-se possível uma definição mais adequada de prioridades e estratégias de prevenção em saúde do trabalhador. Entretanto, ainda existem muitos desafios a serem superados.

Dentre os desafios apontados pela literatura, têm-se a pouca fidelidade dos sistemas de informação em saúde proveniente da má qualidade dos dados, seja pela falha no preenchimento dos campos, seja pela dualidade ou inconsistência dos dados (GOMES SCS e CALDAS AJM, 2017). Outro desafio enfatizado relaciona-se ao levantamento incorreto dos dados, uma vez que nem todas as empresas notificam quando acontecem acidentes cujo afastamento do empregado seja inferior a 16 dias (BATISTA AG, et al., 2019; GOMEZ CM, et al., 2018).

Durante a explanação das temáticas que foram contempladas no evento, a dificuldade dos profissionais em saberem como preencher e os agravos que devem ser notificados emergiu como um aspecto importante a ser discutido. Quando essas dúvidas não são sanadas, abre viés para uma formação deficiente, culminando em uma assistência fragilizada. Sendo assim, isso repercutirá de forma negativa na qualidade e quantidade dos registros, no planejamento e execução das ações, rompendo com a proposta de integralidade do cuidado referida pelo SUS e requerida pela saúde do trabalhador.

Nessa perspectiva, é fundamental que os profissionais da saúde detenham conhecimentos para prestar um cuidado integral aos trabalhadores, bem como realizar o devido registro em situações de danos e/ou óbitos relacionadas ao trabalho, pois estes contribuem para delinear o perfil da ST e assim desenvolver ações voltadas à problemática (ROLOFF DIT, et al., 2016; FERREIRA MJM, et al., 2017).

A baixa adesão dos profissionais de saúde que atuam na assistência ao evento, sinaliza a significativa necessidade de sensibilização que estes precisam ter em relação a saúde do trabalhador. De acordo ao estudo de Gomez CM, et al. (2018) o fato de alguns profissionais não participarem desses momentos parte de um aspecto cultural onde, desde a década de 90 , surgiram alguns entraves para a consolidação da saúde do trabalhador no âmbito da saúde pública, dentre eles, resistência das vigilâncias tradicionais a incorporar o binômio saúde/ trabalho em suas práticas. 
A falta de adesão dos trabalhadores em processos de discussão e formação em ST justificam as dificuldades que eles encontram no desconhecimento e/ou inaptidão no preenchimento das fichas de notificação, pois, ciente da sobrecarga de trabalhos e demandas no serviço público de saúde, as ações de educação permanente em saúde são realizadas com esse intuito, orientar o profissional da condução adequada de sua assistência.

Sendo a Atenção Primária a Saúde (APS) a porta de entrada do usuário no serviço público de saúde, é uma competência dos seus profissionais, realizar ações de saúde para promover a saúde do trabalhador, bem como referenciar os casos de maiores complexidades as instituições especializadas, realizar a notificação nos respectivos sistemas de informação, além de fazer a investigação dos ambientes de trabalho, a fim de verificar se os mesmos apresentam riscos à saúde do operário, e, sempre que possível discutir com o trabalhador as principais causas do seu adoecimento e traçar junto a ele um plano de cuidados (SOUZA TS e VIRGENS LS, 2013).

Silva A, et al. (2016) afirmam que a introdução da ST no setor público tem se disposto um desafio duradouro para o avanço técnico-operacional das práticas de saúde. Esse desafio pode ser visto em todos os níveis de atenção como na assistência, na vigilância à saúde, no manuseio das informações, na gestão dos serviços, na administração de políticas locais e programáticas, e na formação e ensino permanente dos profissionais da saúde. Por isso, há a premência de aplicações congruentes em qualificação dos trabalhadores que nutrem os sistemas de informação, e supervisão sistemática da geração de informação, do colhimento até a crítica e disseminação dos dados, visando a qualidade dos registros dos fatos (GALDINO A, et al., 2016; PIMENTA GR, et al., 2017).

As ações de capacitação dentre outras atividades, como o I Seminário de Saúde do Trabalhador propiciam espaços de discussão, troca de experiências, sensibilização e aprendizado. É durante a formação que o profissional tem suas primeiras vivências no cenário do trabalho em saúde, e é ainda nesse contexto que devem ser afloradas as competências essenciais para a atuação no contexto da saúde do trabalhador. A aproximação prévia do estudante com as problemáticas do ambiente laboral proporciona o desenvolvimento de habilidades no momento em que for trabalhador e /ou coordenador dos serviços de saúde.

Nesse sentido, Faria MGA, et al. (2016) afirmam que a formação do discente precisa ser fundamentada em propostas metodológicas que comportem o desenvolvimento crítico e intelectual, como a problematização. As ações baseadas nesta perspectiva permite aos estudantes elaborarem reflexões críticas ancoradas no conhecimento científico e relacionando-o às práticas cotidianas, que envolvem os sujeitos, modo de vida, e 0 mundo do trabalho, de forma a perceber as implicações desses fatores para as condições de saúde dos trabalhadores.

O seminário foi uma estratégia adotada para aproximar os discentes da ST e discutir temas relevantes na área, haja vista que é a disciplina que envolve muitas determinações políticas, o que desperta pouco interesse do acadêmico, se tornando um desafio a ser contornado pelo docente. Quando realizamos um evento no âmbito da graduação em enfermagem que propõe a discussão da ST com ênfase nos agravos, notificações e sistemas de informação, consequentemente promovemos ampliação da formação dos (futuros) profissionais e fortalecimento do Sistema Único de Saúde. Por meio do I Seminário de Saúde do Trabalhador pôde-se evidenciar a carência de diálogos voltados a ST, bem como as lutas para a consolidação das ações nessa esfera, em destaque o baixo interesse dos profissionais de saúde em se aperfeiçoarem na área, o que gera dificuldades em manejar os sistemas de informação. O evento constitui-se como uma experiência exitosa de construção coletiva e compartilhada, pois colocou a saúde do trabalhador no centro dos debates, contribuindo na formação dos estudantes e fomentando novas discussões acerca da temática dentro da universidade.

\section{REFERÊNCIAS}

1. BRASIL. Ministério da Saúde. Secretaria de Atenção à Saúde. Departamento de Ações Programáticas Estratégicas. Notificação de acidentes do trabalho fatais, graves e com crianças e adolescentes. Brasília (DF): Ministério da Saúde; 2006.

2. BATISTA AG, et al. Registro de dados sobre acidentes de trabalho fatais em sistemas de informação no Brasil. Ciênc. saúde coletiva, 2019; 24(3): 693-704. 
3. FARIA MGA, et al. Inserção de graduandos de referência em serviços de saúde do trabalhador: relato de experiência. Cogitare Enfermagem. 2016; 21(4).

4. FERREIRA MJM, et al. Vigilância dos acidentes de trabalho em unidades sentinela em saúde do trabalhador no município de Fortaleza, nordeste do Brasil. Ciênc. saúde coletiva, 2017; 22(10): 3393-3402.

5. FRANZ EC, CARGNIN MCS. Agravos relacionados com o trabalho notificados no sistema de informações em saúde do trabalhador. Cogitare Enferm., 2018; 23(2): e52345.

6. GALDINO A, et al. Qualidade do registro de dados sobre acidentes de trabalho fatais no Brasil. Rev Saúde Pública, 2017; 51:120.

7. GOMES SCS, CALDAS, AJM. Qualidade dos dados do sistema de informação sobre acidentes de trabalho com exposição a material biológico no Brasil, 2010 a 2015. Rev Bras Med Trab., 2017; 15(3): 200-208.

8. GOMEZ CM, et al. Saúde do trabalhador: aspectos históricos, avanços e desafios no Sistema Único de Saúde. Ciênc. saúde coletiva, 2018; 23(6): 1963-1970.

9. LIMA RKS, et al. Notificação compulsória de acidentes de trabalho: dificuldades e sugestões dos profissionais de saúde em Fortaleza, Ceará. Revista Brasileira de Medicina do Trabalho. 2018; 16(2): 192-

10. International Labour Office (ILO). Word employment and social outlook: Trends 2015. Geneva: ILO, 2015; 100p.

11. Pedroso HC, Gonçalves CGO. Percepção e conhecimento dos profissionais da saúde da atenção primária sobre notificação da perda auditiva induzida pelo ruído em Curitiba - Paraná. CoDAS, 2016; 28(5): 575-582.

12. PIMENTA GRP, et al. Ações de promoção e prevenção à saúde do trabalhador sob risco de exposição e transmissão de hepatites virais. Rev. APS, 2017; 20(1): 140-144.

13. ROLOFF DIT, et al. Enfermeiros do trabalho: experiência interdisciplinar em saúde do trabalhador. Rev Bras Enferm., 2016; 69(5): 897-905.

14. SILVA A, et al. Ações em Saúde do Trabalhador desenvolvidas na Atenção Primária no município de Chapecó, Santa Catarina. Rev. bras. saúde ocup., 2016; $41: e 16$.

15. SOUZA TS, VIRGENS LS. Saúde do trabalhador na Atenção Básica: interfaces e desafios. Rev. bras. saúde ocup., 2013; 38(128).

16. VASCONCELLOS, L. C. F; AGUIAR, L. Saúde do Trabalhador: necessidades desconsideradas pela gestão do Sistema Único de Saúde. Saúde debate, 2017; 41(113): 605-617. 